\title{
Feocromocitoma con trombo en cava
}

\author{
J. López Ferrandis, A. Saiz Sansi, M. Cervera Delgado*, J. Rioja Zuazu, \\ J.M ${ }^{a}$. Regojo Balboa, J.M. Berián Polo
}

Departamento de Urología. *Departamento de Cirugía General. Clínica Universitaria de Navarra. Pamplona.

Actas Urol Esp 2005; 29 (3): 281-286

\section{RESUMEN}

FEOCROMOCITOMA CON TROMBO EN CAVA

El feocromocitoma, paraganglioma de localización suprarrenal, es un tumor cromafín secretor de catecolaminas. La extensión de éstos tumores a vena cava es rara y que el trombo alcance la aurícula derecha es excepcional. Presentamos el caso de una paciente que, sin clínica previa, presentó un cuadro de disfunción multiorgánica como primera manifestación de un tumor suprarrenal con extensión vascular hasta la aurícula derecha y afectación de la vena suprahepática derecha.

Palabras clave: Feocromocitoma. Trombo tumoral. Tratamiento quirúrgico.

\section{ABSTRACT}

\section{PHEOCHROMOCYTOMA WITH THROMBUS IN CAVA}

Pheochromocytoma, a paraganglioma of suprarenal location, is a catecholamine-secreting chromaffin cell tumour. Spread of these tumours to the vena cava is rare and the thrombus only reaches the right atrium in exceptional cases. We present the case of a patient who, without previous symptomatology, presented with a clinical picture of multiorganic dysfunction with primary manifestation of a suprarenal tumour with vascular spread to the right atrium affecting the right suprahepatic vein.

Keywords: Pheochromocytoma. Tumoural thrombus. Surgical treatment.

$\mathrm{E}$ 1 feocromocitoma es un tumor cromafin secretor de catecolaminas, cuyo origen es generalmente la médula adrenal. Este tipo de tumores raramente se extiende a la vena cava. La experiencia quirúrgica en los casos de tumor renal con trombo en cava ha dado lugar al desarrollo de técnicas quirúrgicas que permiten la exéresis del tejido tumoral cuando no existe enfermedad metastásica y constituyen la única alternativa de supervivencia en estos pacientes. Presentamos el excepcional caso de un feocromocitoma maligno con trombo en cava hasta aurícula derecha.

\section{CASO CLÍNICO}

Paciente de 45 años de edad, sin antecedentes personales de interés a excepción de palpitaciones ocasionales. Presentó de forma súbita un cuadro de vómitos y malestar general sin fiebre, tos ni disnea, por lo que acudió al Servicio de Urgencias donde fue diagnosticada de neumonía e ingresada para iniciar tratamiento antibiótico. A las 24 horas del ingreso se agravó el cuadro con fiebre, hipoxemia progresiva y oliguria, por lo que fue trasladada a la unidad de cuidados intensivos (UCI).

Al ingreso en la UCI presentaba una frecuencia respiratoria mayor de 40 , saturación de oxígeno del $85 \%$ con reservorio, frecuencia cardiaca de 140 ppm, tensión arterial 120/40 mmHg, temp-35으. La auscultación cardiopulmonar mostraba crepitantes en ambos hemitorax y la exploración física destacaba ausencia de pulsos distales aunque sin edemas en extremidades. En la analítica realizada destacaba leucocitosis de 
36400, Glucosa: $266 \mathrm{mg} / \mathrm{dL}$, Creatinina: 3,3 mg/dL, AST: 3127 U/L, ALT: 3139 U/L, Gamma GT: 86 U/L y tiempos de coagulación ligeramente elevados. Gasometría: $\mathrm{PH}: 7,11 . \mathrm{PCO}_{2}: 41 . \mathrm{PO}_{2}$ : 44. Saturación $\mathrm{O}_{2}: 63$.

En la Radiografía de tórax se apreciaba infiltrado alveolo-intersticial bilateral de predominio derecho. Se procedió a la intubación inmediata y se inició tratamiento con antibióticos de mayor espectro. La paciente presentó un cuadro de fracaso multiorgánico y en una nueva analítica llamó la atención la elevación de la creatinin-fosfoquinasa y troponina, por lo que se realizó ecocardiograma transesofágico, objetivándose masa a nivel de cava superior que llegaba hasta la aurícula derecha. Se realizó una tomografía computerizada (TC) que informó de derrame pleural bilateral sin tromboembolismo pulmonar, dilatación y ocupación de la vena cava inferior intrahépatica que proximalmente llega hasta la aurícula derecha y distalmente hasta las venas renales. Hipodensidad de la vena suprahepática derecha compatible con trombosis secundaria y masa suprarrenal derecha de $4 \mathrm{~cm}$ hipercaptante, en intimo contacto con la vena cava inferior. (Figuras 1 y 2)

Habiendo estabilizado a la paciente fué remitida a la UCI de nuestro centro a los 4 días de su ingreso, intubada y con disfunción multiorgánica, con el fin de planificar tratamiento quirúrgico.

Se repite la analítica en la que destaca leucocitosis, AST: 165 UI/L, ALT: 1050 UI/L con ionograma y creatinina normales. Tras valorar las pruebas aportadas, dada la gravedad del cuadro,

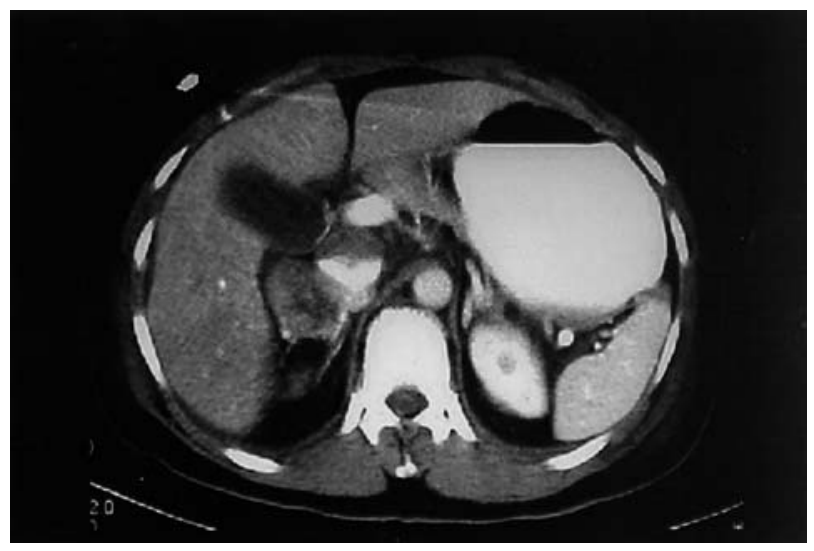

FIGURA 1. Tumor suprarrenal en intimo contacto con vena cava y ocupación de la misma por trombo tumoral.

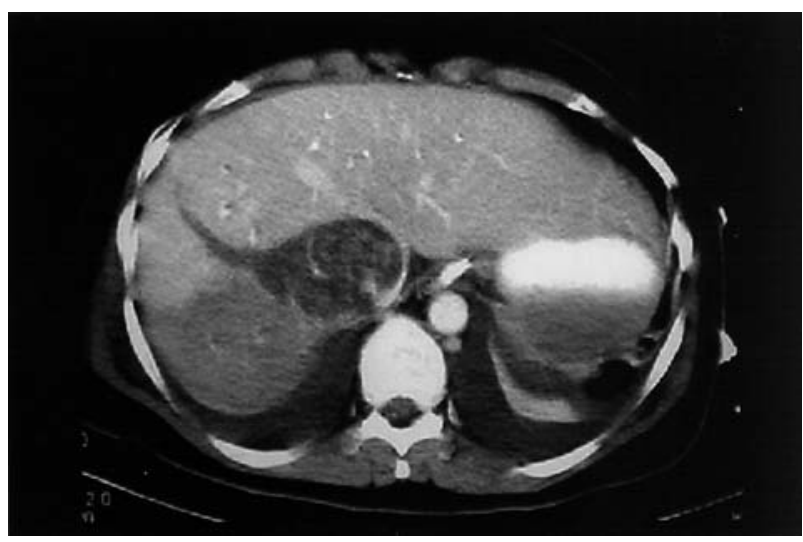

FIGURA 2. Trombo en vena cava con ocupación de vena suprahepática derecha e isquemia secundaria de los segmentos hepáticos VI y VII.

y puesto que las pruebas de imagen realizadas no presentan metástasis a distancia, se planteó un tratamiento quirúrgico urgente.

Se realiza una laparatomía subcostal bilateral al mismo tiempo que una esternotomía longitudinal. Se aprecia derrame pleural bilateral, ligero derrame pericárdico y corazón con contracción normal en la cavidad torácica, mientras que la laparotomía muestra isquemia de los segmentos 6 y 7 del hígado y masa suprarrenal derecha.

El equipo de Cirugía General realiza hepatectomía anterior con maniobra de Pringle intermitente, sin voltear el hígado, seccionando el hígado hasta llegar a cara anterior de la Cava retrohepática. Posteriormente, el equipo de Urología tras descolgar el colon derecho realiza disección de vena cava hasta iliacas primitivas, ligadura de vena genital derecha, liberación de vena renal y cara anterior de riñón derecho. Se procede a la liberación de la glándula suprarrenal derecha, a excepción de su borde superior y medial que está intimamente unido a la pared derecha de la vena cava. Queda así expuesto el campo para la exéresis de suprarrenal e hígado derecho a falta de cavotomía. (Figura 3A).

Una vez realizado esto, el equipo de Cirugía Cardiaca comienza la circulación extracorpórea bajando progresivamente la temperatura hasta alcanzar $14^{\circ} \mathrm{C}$ a nivel esofágico y $19^{\circ} \mathrm{C}$ a nivel rectal, tras lo cual se procede a exanguinar a la paciente y parar la circulación extracorpórea manteniendo la sangre en un oxigenador. Es entonces cuando se procede a realizar una cavo- 


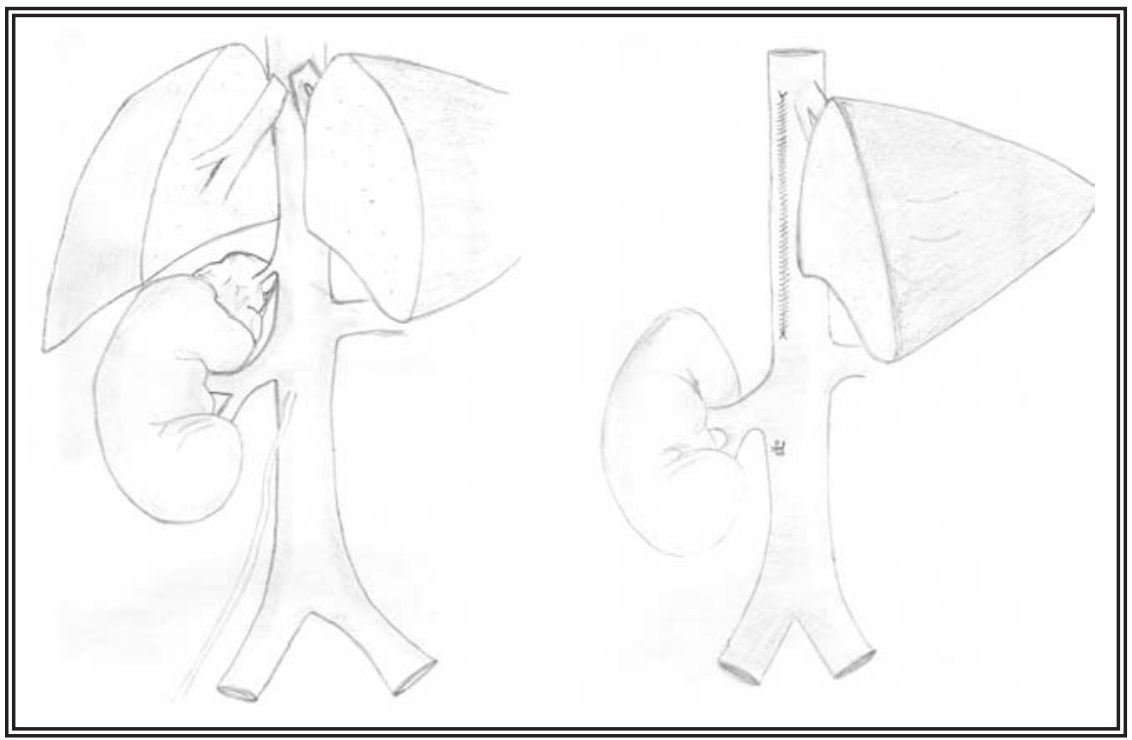

FIGURA 3. Esquema de la exposición del campo quirúrgico.

A) Previo al inicio de circulación extracorpórea. Resección de segmentos VI y VII hepáticos, liberación de riñón y suprarrenal derecha asi como vena cava desde diafragma hasta bifurcación ilíaca.

B) Campo quirúrgico tras la exéresis de masa suprarrenal y segmentos hepáticos VI y VII.

tomía inferior a nivel de suprarrenal hasta $2 \mathrm{~cm}$ de diafragma, aurículotomía derecha y retirada del trombo tumoral a través de la incisión realizada en la cava inferior. Se realiza sección de pared lateral derecha de vena cava (desde la vena suprarrenal hasta la vena suprahepática derecha), extrayendo en bloque hígado derecho con venas suprahepática y suprarrenal derechas incluidas en dicho parche, suprarrenal derecha y trombo. Se produce rotura del trombo a nivel de vena renal, por lo que en un segundo tiempo se extrae por tracción el trombo desde vena renal hasta iliacas primitivas. Se realiza irrigación de cava y aurícula derecha y, al no observarse restos de trombo ni infiltración de las paredes de cava o aurícula, se procede al cierre de vena cava y orejuela derecha por separado y a la vez mediante sutura continua con monofilamento (Figuras 3B y 4). Una vez realizado dicho cierre se procede a reperfundir progresivamente a la paciente volviendo a entrar en circulación extracorpórea, recalentándola y procediendo a desclampar la aorta y perfundir el corazón al alcanzar una temperatura esofágica de $34^{\circ} \mathrm{C}$. El inicio de la función cardiaca permite la salida de circulación extracorpórea.

Se colocan drenajes retroesternal, mediastínico, subhepático y retroperitoneal en fosa renal derecha. Durante la intervención se transfundieron 14 concentrados de hematíes, $1200 \mathrm{ml} \mathrm{de}$ plasma y 2 unidades de plaquetas. Durante la estancia en la UCI se realiza ecografía abdominal que muestras buena permeabilidad del sistema venoso hepático izquierdo y de vena cava. A los 10 días la paciente es trasladada a planta, encontrándose consciente, orientada y colaboradora, aunque presentando debilidad en las cuatro extremidades. La paciente permanece ingresada en planta durante 20 días recibiendo tratamiento de cinesiterapia generalizada pasiva y asistida. Tras buena evolución en la movilidad es dada de alta, no precisando tratamiento a excepción de heparina de bajo peso molecular.

La anatomía patológica informa de feocromocitoma que afecta a la glándula suprarrenal derecha cuyo diámetro mayor es de $2 \mathrm{~cm}$. El

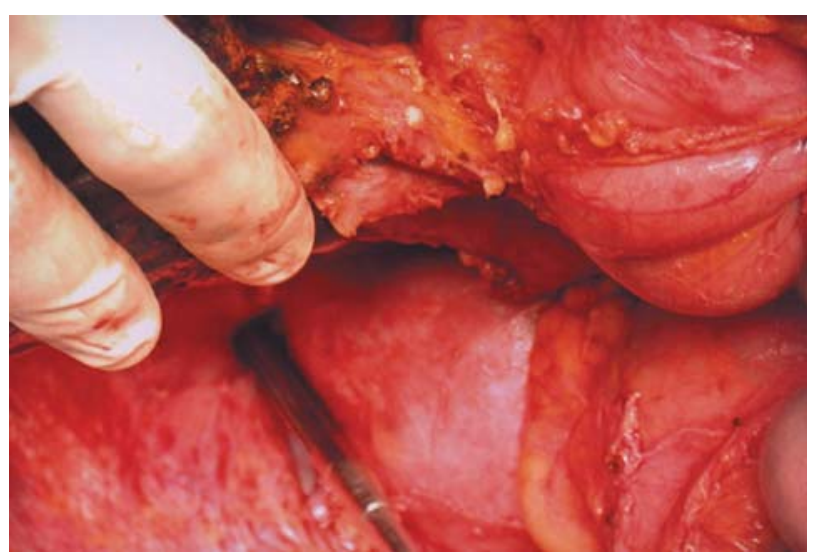

FIGURA 4. Campo quirúrgico tras la exéresis de masa suprarrenal y segmentos hepáticos VI y VII. Se aprecia polo superior de riñón derecho, higado izquierdo y sutura en vena 
tumor no está encapsulado y se extiende fuera de la glándula suprarrenal, sin afectar a los órganos adyacentes. Existe necrosis tumoral extensa. La glándula suprarrenal no neoplásica no muestra anomalías. Los ganglios linfáticos estudiados no presentan tumor. En el parénquima hepático hay extensas áreas de hemorragia centrolobulillar, con necrosis de hepatocitos sin observarse células tumorales en los fragmentos estudiados del parénquima hepático. Trombo infrarrenal hemático, sin evidencia de células tumorales.

En la revisión realizada a los 2 meses, la paciente se encontraba asintomática, realizando vida activa y la TC no mostró lesiones sugestivas de enfermedad tumoral.

\section{COMENTARIOS}

El feocromocitoma, paraganglioma de localización suprarrenal, es un tumor de tejido cromafin secretor de catecolaminas. La mayor parte de tejido cromafin se encuentra en la médula adrenal y el resto es tejido extradrenal paraganglionar simpático. La mayoría son esporádicos, tan solo un $10-20 \%$ son hereditarios ${ }^{1}$. Es un tumor infrecuente, que tan solo aparece en el 0,3\% de los pacientes evaluados por hipertensión ${ }^{1} \mathrm{y}$ en un $0,05 \%$ de las autopsias ${ }^{2}$. Puede ocurrir a cualquier edad siendo más frecuente hacia la cuartasexta década ${ }^{1}$, no habiendo grandes diferencias entre sexos.

El $60-70 \%$ de los paragangliomas son funcionantes ${ }^{3}$ y sus manifestaciones clínicas son variables, siendo la hipertensión arterial (HTA) el signo más frecuente generalmente como HTA mantenida y en otros casos como crisis o paroxismos hipertensivos. La HTA puede ser maligna y resistente al tratamiento convencional. Los sintomas más frecuentes son dolor de cabeza, palpitaciones, hipotensión ortostática, ansiedad, sudoración profusa, dolor abdominal, dolor torácico, temblor o visión borrosa. Otros datos que pueden aparecer son: disminución del volumen circulante con elevación de las cifras de hematocrito y hemoglobina (por vasoconstrición vascular), hiperglucemia, hipercalcemia, fiebre, rabdomiólisis con insuficiencia renal mioglobinúrica (isquemia muscular por vasoconstricción), colelitiasis, angina, infarto, arritmias, taquicardias ${ }^{1,4,5}$.
La elevación de los niveles de metanefrinas, norepinefrinas y epinefrinas en orina de 24 horas tiene una sensibilidad diagnóstica del 89,9\%3․

Las pruebas de imagen más utilizadas para su localización son la TC, con una eficacia diagnóstica del $89-96 \%$ y la resonancia magnética (especificidad del $67 \%$ y sensibilidad del $100 \%$ en feocromocitomas adrenales), apareciendo típicamente hiperintensos en $\mathrm{T}_{2}^{4}$. La gammagrafia con metayodobenzilguanidina presenta una sensibilidad del $88 \%$ con una especificidad del $100 \%$ por lo que es muy útil para detectar metástasis, tumor residual postcirugia, recidiva local o paragangliomas extraadrenales ${ }^{4}$. Para la detección del trombo tumoral, la resonancia magnética puede ser más precisa que la TC, presentando una sensibilidad entre el $80-100 \%$, superior a la TC y similar a la cavografía. A pesar de todo, actualmente es la ecografía mediante la utilización del Doppler-color la técnica de elección para el diagnóstico de trombo tumoral, al detectar flujo arterial en el interior del trombo ${ }^{1}$. Cuando el trombo tumoral ocluye totalmente la luz de la cava se puede formar una trombosis venosa no tumoral, que puede llegar a superar la bifurcación iliaca, siendo raro que el trombo tumoral progrese caudalmente hacia los vasos iliacos ${ }^{7}$.

E1 $72-82 \%$ de los paragangliomas se sitúan en una única glándula suprarrenal, el 3-11\% son bilaterales y en torno al 9-19\% son extraadrenales ${ }^{1}$. Alrededor del 4-14\% son malignos ${ }^{8}$. La diferencia de malignidad o benignidad no depende de parámetros histológicos, sino por la presencia de metástasis o la invasión de tejidos vecinos ${ }^{1,8}$. La definición de enfermedad metastásica está basada en la presencia de tumor en localizaciones anatómicas donde la existencia de tejido cromafin no es normal. Los paragangliomas metastatizan en un $20-40 \%$ de los casos, siendo el hueso la localización más frecuente seguido de hígado, pulmón y cerebro ${ }^{3}$. El feocromocitoma raramente se extiende dentro de la vena cava. Hartgrink y cols $^{9}$ en una revisión realizada en 2001 encuentran unos 20 casos y sólo 3 con extensión hasta la aurícula derecha, tratándose en dos casos de feocromocitomas resecados previamente, encontrado un único caso de feocromocitoma primario con afectación de la vena cava y aurícula derecha). Por nuestra parte, no hemos encontrado 
ningún otro caso de feocromocitma primario con trombo tumoral hasta aurícula derecha. El tratamiento electivo de estos tumores consiste en la extirpación quirúrgica debido a la clínica, sobre todo por la hipertensión arterial que producen y a la posibilidad de malignización ${ }^{3}$. La quimioterapia con ciclofosfamida, vincristina y dacarbazine tiene una respuesta bioquímica del $79 \%$ y del tumor del $57 \%$, con una duración de respuesta limitada y la radioterapia es utilizada de modo paliativo en metástasis óseas con resultados satisfactorios ${ }^{1}$.

El tratamiento de los tumores con afectación vascular sigue siendo controvertido. En los casos de tumor renal, parece que la presencia del trombo cuando no existe afectación de la pared del vaso, por si misma no marca el pronóstico. En estos casos, la frecuente asociación de afectación ganglionar y/o la presencia de metástasis a distancia es la que ensombrece el pronóstico ${ }^{10}$. En un estudio realizado por nuestro grupo ${ }^{11}$ de los tumores renales pT3, se concluye que el subestadio (pT3a, pT3b, pT3c) no es factor independiente de progresión de la enfermedad ni influye en la supervivencia global y que los pacientes con invasión vascular tienen un mayor porcentaje de recidivas, aunque no se asocia a una menor supervivencia.

Desde el trabajo de Skiner y $\operatorname{cols}^{12}$ en 1971 demostrando mejor supervivencia en los pacientes con carcinoma renal en los que se realiza tratamiento agresivo del trombo tumoral en la vena cava, se ha producido un gran cambio en el tratamiento de éstos tumores. Es importante conocer si el trombo tumoral llega o no a la altura de las venas suprahepáticas, no por factor pronóstico sinó para establecer una estrategia de traba$\mathrm{jo}^{13}$. En tumores con afectación infrahepática de la cava, la vía anterior con cavotomía y cierre directo o con parche sintético suele ser suficiente. Cuando la afectación es suprahepática, requiere en nuestra experiencia la colaboración de cirujanos cardiacos y abordaje auricular y abdominal, con derivación extracorpórea e hipotermia. Es una cirugía de riesgo con elevada morbilidad $^{14}$. Cuando el trombo tumoral sobrepasa el trayecto retrohepático de la cava, si no existen metástasis, está indicado el tratamiento ${ }^{15}$. El procedimiento que consideramos más adecuado es el descrito por Marshall en $1984^{16}$. Se realiza hipotermia con parada cardiaca, almacenando temporalmente la sangre, con bypass cardiopulmonar. De esta forma se crea un campo limpio de sangre que permite la extracción del carcinoma renal con trombo tumoral hasta la aurícula. Una vez expuesto el campo es el momento de comenzar el bypass cardiopulmonar y se va enfriando al paciente hasta alcanzar $19.5^{\circ} \mathrm{C}$. La cabeza es envuelta en hielo. Se para la bomba del bypass y la sangre del paciente es extraida y mantenida en el oxigenador. Es en este momento cuando se puede incidir la vena cava desde la salida de la vena renal hasta las hepáticas. Se abre a la vez la aurícula y se extrae el trombo. Se debe de irrigar la cava y revisar las venas suprahepáticas y la renal contralateral, extrayendo los restos del trombo si los hubiera. Una vez suturada la vena cava y la aurícula derecha, se vuelve a poner en marcha el bypass, recuperando la actividad cardiaca y pulmonar.

La ocupación de las venas suprahepáticas es un factor pronóstico ominoso. Cuando esta oclusión produce el síndrome de Budd-Chiari, es de gran importancia el tratamiento quirúrgico inmediato ${ }^{17}$, con o sin resección hepática ${ }^{18}$. El síndrome de Budd-Chiari es una forma poco frecuente de hipertensión portal cuya etiología es desconocida (predisponen situaciones de hipercoagulabilidad). En nuestro caso se debe a la ocupación masiva de la vena cava por el trombo tumoral. La forma de presentación variará según el número de venas hepáticas afectas, grado de obstrucción y rapidez de instauración, la evolución natural es a la destrucción del hígado y el pronóstico es imprevisible.

En el caso que nos ocupa, no se realizaron las pruebas diagnósticas oportunas para valorar el tipo de tumor suprarrenal ni tampoco otras exploraciones complementarias además de la TC, de ayuda para la valoración de metástasis, puesto que la situación de la paciente era de un cuadro de aparición brusca y con un pronóstico ominoso si no se realizaba un tratamiento quirúrgico urgente.

Nuestra actitud en pacientes de régimen ambulatorio con tumores, sobre todo renales, con afectación vascular es descartar la presencia de metástasis a distancia y tratamiento quirúrgico agresivo siempre que no exista evidencia de enfermedad en otros órganos. 


\section{REFERENCIAS}

1. Walther MM, Keiser HR, Linehan. WM Pheocromocytoma: evaluation, diagnosis and treatment. World J Urol 1999; 17:35-39.

2. McNeil AR, Blok BH, Koelmeyer TD, Burke MP, Hilton JM. Pheocromocytomas discovered during coronial autopsies in Sydney, Merlbourne and Auckland. Aust N Z J Med 2000; 30:648-652.

3. Cervera M, Olea JM, Diez-Caballero A, et al. Paraganglioma extraadrenal localizado por PET y extirpado por vía laparoscópica. Cir Esp 2003;73(2):138-140.

4. Foggo J. Phaeocromocytoma: a case study. Clinical Chiopractic. 2003;6:50-54.

5. Vierhapper H. Adrenocortical tumors: clinical symptoms and biochemical diagnosis. European Journal of Radiology. 2002;41:88-94.

6. Sánchez D, López J, Arocena J, et al. Clínica, diagnóstico y pronóstico del carcinoma renal. Actas Urol Esp 2002; 26(8): 532-540.

7. Giuliani L. Atlas of surgery for renal cancer. Second edition. Lo Zambaletti, SpA 1991.

8. De Toma G, Letizia C, Cavallaro G, et al. Malignant pheocromocytoma. Personal experience, review of the literature. Ann Italiani di Chirugia. 2002;73(4):413-8.

9. Hartgrink HH, Roelfsema F, RAEM Tollenaar, PAE Hiddema, MEJ Pijl, CJH Velde. Primary pheocromocytoma extending into the right atrium: report of a case and review of the literature. European Journal of Surgical Oncology, 2001; 27(1):115-119.

10. Mejean A, Ouard S, Thiounn N. Prognosis factors of renal cell carcinoma. J Urol 2003;169:821-827.

11. Sánchez D, Arocena J, Regojo JM, et al. Factores pronósticos en el carcinoma renal pT3. Actas Urol Esp 2003; 27 (1):26-32.
12. Skiner DG, Colvin RB, Vermillion CD, Pfister RC, Leadbetter WF. Diagnosis and management of renal cell carcinoma. A clinical pathological study of 309 cases. Cancer 1971;28:1165.

13. Belis JA, Pae WE, Rhoner TJ, et al. Cardiovascular evaluation before circulatory arrest for removal of vena caval extension of renal cell carcinoma. J Urol,. 1998; 141:1302.

14. Arocena J, López J, Sánchez D y cols. Tratamiento del carcinoma renal. Actas Urol Esp 2002; 26(8):541-545.

15. Rodríguez-Rubio FI, Abad JI, Sanz G y cols. Surgical management of retroperitoneal tumors with vena caval thrombous in the inferior cava using cardiopulmonary bypass, arrested circulation and profound hypotermia. Eur Urol 1997;32(2):194-197.

16. Marshall FF, Reitz BA, Diamond DA. A new technique for management of renal cell carcinoma involving the right atrium: hypotermia and cardiac arrest. J Urol. 1984; 131: 103.

17. Ciancio G, Soloway M. Renal cell carcinoma invading the hepatic veins. Cancer 2001;92(7):1836-1842.

18. Sener SF, Winchester DJ, Votapka TV, McGuire MS $\mathrm{O}^{\prime}$ Connor B, Szokol JW. Continuing experience with liver resection and vena cava reconstruction using cardiopulmonary bypass and hypotermic circulatory arrest. Am Surg 2002;68(4):359-63.

Dr. J. López Ferrandis

Clínica Universitaria de Navarra

Servicio de Urología

Apartado 4209

31080 Pamplona (Navarra)

(Trabajo recibido el 6 junio de 2004) 